\title{
Osteoblastoma Mimic Failed Back Surgery
}

\author{
Juan F. Blanco ${ }^{1}$, Susana Gomez-Castro ${ }^{2}$, Ruth Lopez-Gonzalez ${ }^{2}$ Juan C. Paniagua ${ }^{3}$ \\ ${ }^{1}$ Department of Trauma and Orthopedics, Hospital Universitario de Salmanca, Salamanca, Spain \\ ${ }^{2}$ Department of Rheumatology, Hospital Universitario de Salmanca, Salamanca, Spain \\ ${ }^{3}$ Department o Radiology, Hospital Universitario de Salmanca, Salamanca, Spain \\ E-mail: juanfblanco@telefonica.net; susigom888@yahoo.es; ruthielg@hotmail.com; ruthielg@hotmail.com \\ Received October 25, 2010; revised January 10, 2011; accepted April 21, 2011
}

\begin{abstract}
A 44 year-old women with persistent back and irradiate pain after discectomy in L5 - S1 lumbar level presented a osteoblastoma in L4 - L5 level. The osteoblastoma mimic a failed back surgery. She was successfully treated by removing the tumour with instrumented posteroalateral fusion. This case warns people that a new disease (such as a tumour in this case), irrelevant to the original one, may happen simultaneously even if the original one has been successfully treated and should be differentiated from the previous disease or surgical failure and complications.
\end{abstract}

Keywords: Osteoblastoma, Lumber Spine, Surgery, Back Pain, Disc Herniation

\section{Introduction}

Osteoblastoma of the spine is a rare benign bone tumour accounting for less than $1 \%$ of all primary bone tumours [1]. Generally, it affects persons 10 to 40 years of age, with a male to female ratio of approximately 2 to $1[2,3]$. Spinal involvement occurs in $35 \%$ to $45 \%$ of cases. Lumbar localization is frequent. The majority arise from posterior spinal elements: pedicles, lamina and facets $[4,5]$. Clinically the patients with osteoblastoma have pain and stiffness [6,7]. This case is presented because of is rarity and also it happened after spine surgery; this one was the reason of the delay in the diagnosis. The symptoms were attributed to a syndrome of failed back surgery.

\section{Case Report}

44-year-old women were admitted in June 2006 with low back and irradiate pain. The past medical history included: apendectomy, histerectomy and depression. In 2004 a lumbar discectomy was performed because of a slipped disc in L5 - S1 level (Figure 1). After surgery the patients continued with lumbar and irradiate pain. Because of the suspicion of periradicular fibrosis, the patient underwent a Magnetic Resonance Imaging (MRI) (Figure 2). The disc degeneration in the L4 - L5 and L5 S1 levels was considered the aetiology of pain. The symptoms assumed to a failed back surgery and she was remitted to the pain unit. In 2006 when she was admitted to our unit, she has back and irradiate pain. Physical and neurological examination found no abnormalities. Plain radiographs showed degenerative disc disease in L4 - L5 and L5 - S1 levels. A MRI was performed and revealed an inflated focal lesion with a $1.5 \mathrm{~cm}$ diameter located in the right lamina of L4, heterogenic, isointensive relationship to the muscle that through contrast administration presents a discrete highlight signs of paravertebral muscles oedema, adjacent to the lesion with notable contrast (Figure 3). Technetium bone scan showing increased focal uptake in the right side of the L4 - L5 level (Figure 4). Before surgery a computed tomography (CT) was performed and showed a well limited expansible litic lesion in the inferior right lamina of L4 vertebra (Figure 5). Routine laboratory examinations were within normal limits. In a restrospective way, little alterations of the signal in MRI can be seen previous to the appearance of the tumour (Figure 2). Posterior approached was performed with complete excision of tumour. Simultaneously a posterolateral fusion with pedicular screws was performed. Pathologic examination of surgical specimen from patient revealed osteoblastoma (Figure 6). After the surgery the symptoms were relieved.

\section{Discussion}

Osteoblastoma is a uncommon neoplasm that accounts for approximately $3 \%$ of benign and $1 \%$ of all primary 


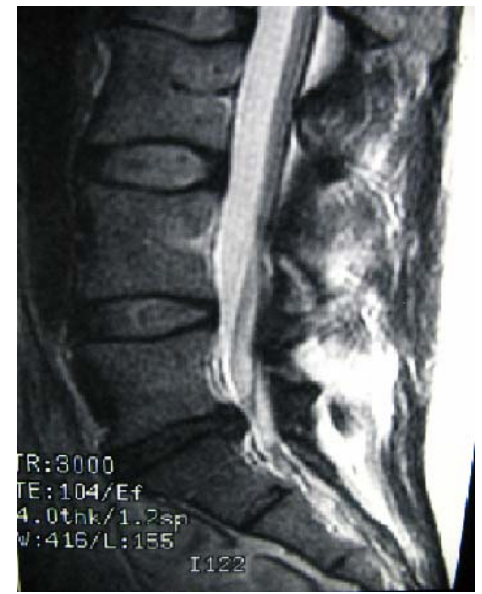

Figure 1. Sagital T2 weighted MRI. Signs of posterocentral slipped disc protude at the L5 - S1 level in the year 2003.



(a)

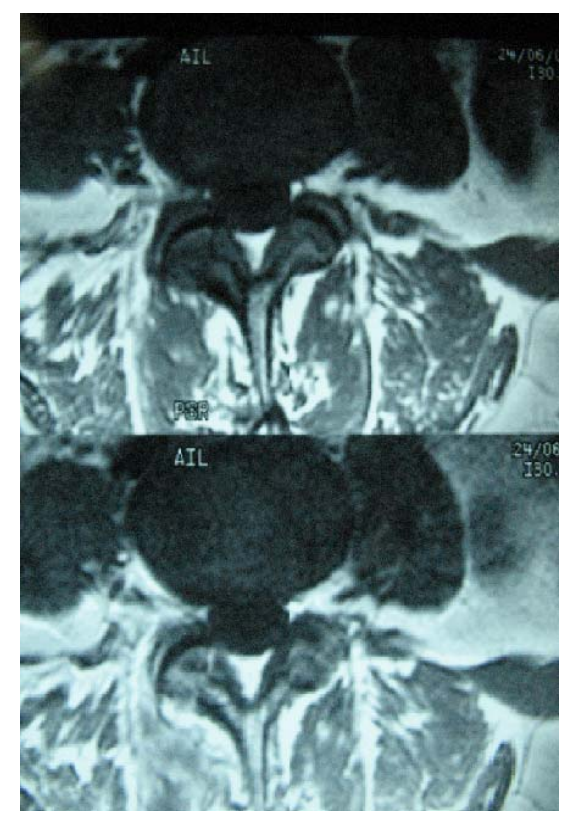

(b)

Figure 2. Axial T2 weighted MRI showing, surgical changes in L5 - S1 level (a) and slight high signal intensity in the facet and lamine of the L4 vertebra on the right side (b). Yellow arrow. (where it wasn't noticed or found important). Year 2004.

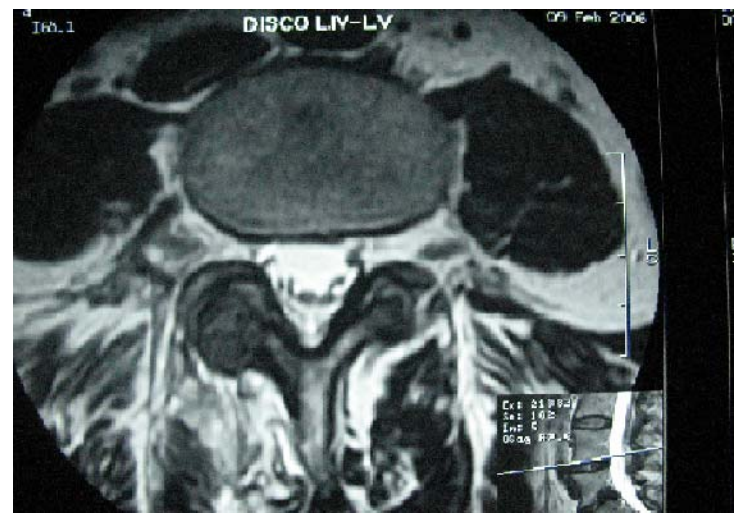

Figure 3. Axial T2 MRI (year 2006) showing expansive lesion and anormal intensity changes in the L4 - L5 right facet. This lesion is heterogenic, isointensive relationship to the muscle that through contrast administration presents a discrete highlight signs of paravertebral muscles oedema, adjacent to the lesion with notable contrast.

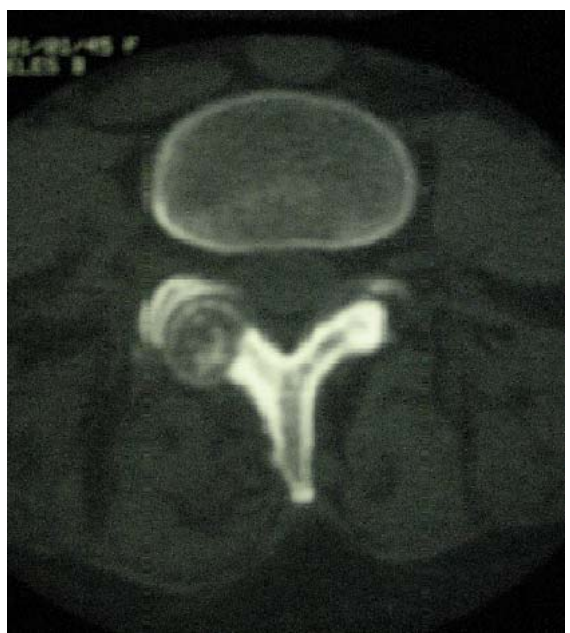

Figure 4. Increase focal uptake in bone scan at the L4 - L5 right level.

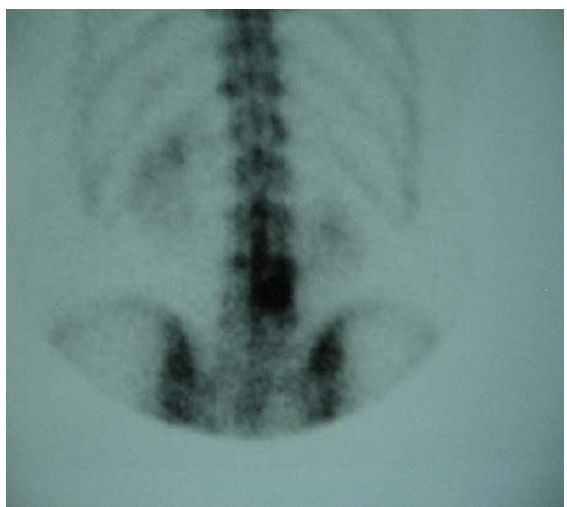

Figure 5. CT showing well-defined litic lesion parcial calcified matrix with a $1.5 \mathrm{~cm}$ diameter located in the right lamina of $\mathbf{L} 4$ and that it is associated with a notable sclerosis and periostotic enlargement of the adjacent bones structures. 


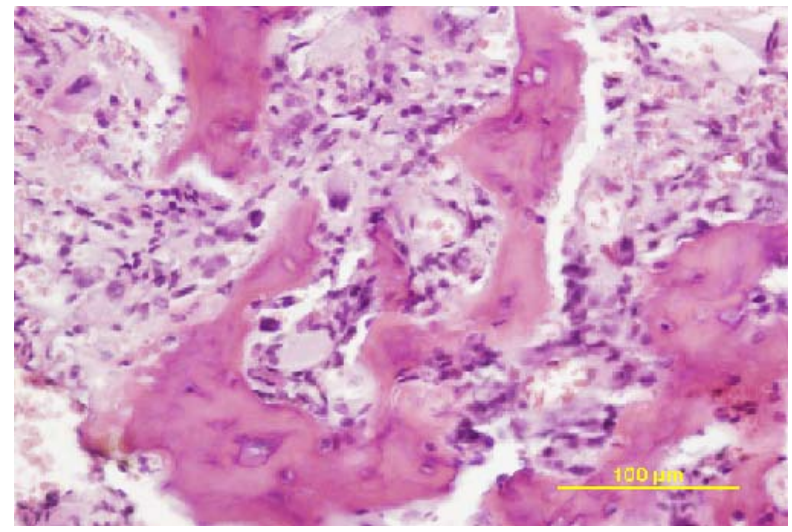

Figure 6. Photomicrograph showing woven bone and osteoid rimmed by osteoblasts within a loose fibrovascular stroma. (Hematoxylin-eosin $\times$ 100).

bone tumors [1]. The spine is the most frequently affected part of the skeleton by osteoblastoma. The majority of tumours arise from posterior spine elements, with coincidence to our case [2-4]. Though the tumour can appear with neurological deficit [2], most patients have located dull pain and tenderness [6]. Osteoblastoma are common cause of painful scoliosis in children and adolescents [8]. The common nature of the presenting symptoms of this entity was thought to be responsible for the delay in their diagnoses before the advent of modern imaging modalities. In this case the history of surgery over an adjacent segment to the lesion lead to a mistaken interpretation delaying diagnosis. The pain was attributed to the failure back surgery.

Osteoblastoma is a solitary lesion. On plain radiographs osteoblastomas plow lucent o lytic, typically expansible, with a lobulated appearance, in which frequently a mineralized matrix exits [6]. When simple radiology is not sufficient the CT or the MRI and gammagraphy can help in the diagnosis. The MRI appearances of spinal osteoblastomas are varied and show no characteristic features [9]. In our case, in a retrospective way, little alterations of the signal in MRI can be seen previous to the appearance of the tumour. These little alterations can be considered as precursors of the osteoblastoma. Emmez et al. [10] have reported a case of a lumbar spinal osteoblastoma with progression and destruction of the disc. In the present case were not observed litics or expansive lesions in the first diagnostic studies.

The treatment is based on the complete extirpation of the tumour to avoid recurrences [6]. In this case the coexistence of a degenerative discopathy and the necessity of extirpate a facet joint lead to the option of spine fusion, in coincidence with other authors [7].

\section{Conclusions}

We report a rare case of spinal osteoblastoma after surgery in adjacent vertebral level. The osteoblastoma mimic a syndrome of failed back surgery which can warn people that a new disease (such as a tumor in this case), irrelevant to the original one, may happen simultaneously even if the original one has been successfully treated and should be differentiated from the previous disease or surgical failure and complications.

\section{References}

[1] D. R. Lucas, K. K. Unni, R. A. McLeod, M. I. O’Connor and F. H. Sim, "Osteoblastoma: Clinicopathologic Study of 306 Cases,” Human Pathology, Vol. 25, No. 2, 1994, pp. 117-134. doi:10.1016/0046-8177(94)90267-4

[2] S. Boriani, R. Capanna, D. Donati, A. Levine, P. Picci and R. Savini, "Osteoblastoma of the Spine," Clinical Orthopaedics, Vol. 5, No. 278, 1992, pp. 37-45.

[3] B. W. Marsh, M. Bonfiglio, L. P. Brady, W. F. Enneking, "Benign Osteoblastoma: Range of Manifestations," Journal of Bone and Joint Surgery, Vol. 57, No. 1, 1975, pp. 1-9.

[4] T. Ozaki, U. Liljenqvist, A. Hillmann, H. Halm, N. Lindner, G. Gosheger and W. Winkelmann, “Osteoid Osteoma and Osteoblastoma of the Spine: Experiences with $22 \mathrm{~Pa}-$ tients," Clinical Orthopaedics, No. 397, 2002, pp. 394402. doi:10.1097/00003086-200204000-00046

[5] Y. Saglik, H. Atalar, Y. Yildiz, K. Basari and C. Gunay, "Surgical Treatment of Osteoblastoma: A Report of 20 Cases,” Acta Orthopaedica Belgica, Vol. 73, No. 6, 2007, pp. 7747-7753.

[6] P. Kan and M. H. Schmidt, "Osteoid Osteoma and Osteoblastoma of the Spine.” Neurosurgery Clinics of North America, Vol. 19, No. 1, 2008, pp. 65-70. doi:10.1016/j.nec.2007.09.003

[7] O. Nemoto, R. P. Moser, B. E. Van Dam, J. Aoki and F. W. Gilkey, "Osteoblastoma of the Spine, a Review of 75 Cases,” Spine, Vol. 15, No. 12, 1990, pp. 1272-1280. doi:10.1097/00007632-199012000-00008

[8] A. Saifuddin, J. White, Z. Sherazi, M. I. Shaikh, C. Natali and A. O. Ransford. "Osteoid Osteoma and Osteoblastoma of the Spine: Factors Associated with the Presence of Scoliosis,” Spine, Vol. 23, No. 1, 1998, pp. 47-53. doi:10.1097/00007632-199801010-00010

[9] M. I. Shaikh, A. Saifuddin, J. Pringle, C. Natali and Z. Sherazi, "Spinal Osteoblastoma: CT and MR Imaging with Pathological Correlation,” Skeletal Radiology, Vol. 28, No. 1, 1999, pp.33-40. doi:10.1007/s002560050469

[10] H. Emmez, M. Kaymaz, N. Tokgöz, G. Yilmaz and G. Kurt, "Progression of a Lumbar Spinal Osteoblastoma," Neurologia Medico-Chirurgica, Vol. 45, No. 7, 2005, pp. 379-383. doi:10.2176/nmc.45.379 